\title{
INTEGRATED DATA CAPTURING REQUIREMENTS FOR 3D SEMANTIC MODELLING OF CULTURAL HERITAGE: THE INCEPTION PROTOCOL
}

\author{
R. Di Giulio ${ }^{\text {a }}$,F. Maietti ${ }^{\text {* }}$, E. Piaia ${ }^{\text {a }}$, M. Medici ${ }^{\text {a }}$ F. Ferrari ${ }^{\text {a }}$, B. Turillazzi ${ }^{\text {b, }}$ \\ ${ }^{a}$ Department of Architecture, University of Ferrara, via Ghiara 36, 44121 Ferrara, Italy - (dgr, mttfrc, piamnl, mdcmrc, \\ frrfrc)@unife.it \\ ${ }^{\mathrm{b}}$ Consorzio Futuro in Ricerca, via Saragat 1, 44122 Ferrara, Italy - tll@ unife.it
}

\section{Commission II}

KEY WORDS: Cultural Heritage, 3D data capturing, Semantic modelling, Data acquisition protocol, Accuracy, Reliability

\begin{abstract}
:
The generation of high quality 3D models can be still very time-consuming and expensive, and the outcome of digital reconstructions is frequently provided in formats that are not interoperable, and therefore cannot be easily accessed. This challenge is even more crucial for complex architectures and large heritage sites, which involve a large amount of data to be acquired, managed and enriched by metadata.

In this framework, the ongoing EU funded project INCEPTION - Inclusive Cultural Heritage in Europe through $3 D$ semantic modelling proposes a workflow aimed at the achievements of efficient 3D digitization methods, post-processing tools for an enriched semantic modelling, web-based solutions and applications to ensure a wide access to experts and non-experts.

In order to face these challenges and to start solving the issue of the large amount of captured data and time-consuming processes in the production of 3D digital models, an Optimized Data Acquisition Protocol (DAP) has been set up. The purpose is to guide the processes of digitization of cultural heritage, respecting needs, requirements and specificities of cultural assets.
\end{abstract}

\section{INTRODUCTION}

\subsection{Research framework}

One of the main current challenges in the creation, management and use of digital models of cultural heritage concerns the dual need for high-quality 3D representations at an affordable cost, easily accessible and reusable. This is one of the main topic launched also by the European Commission within the program Horizon 2020 and in particular in the Societal Challenge Work Programme: to contribute to a deeper awareness and understanding of European cultural heritage as inspiration for addressing contemporary challenges, increasing the knowledge of heritage and its different identities. To meet this purpose, new technologies and digitization processes play a key role since they allow new and enhanced interpretations of our collective cultural heritage.

As stated in the Work Programme, Europe's most pressing challenge is to overcome the current economic crisis; this is why Europe 2020 places research, technology and innovation at the forefront of activities designed to help Europe building smart, sustainable and inclusive growth. New knowledge, technologies and innovations can turn these challenges into opportunities. Moreover, in challenging times for its internal coherence, Europe should improve the understanding of its cultural heritage and of its identities in order to strengthen cohesion and solidarity and to encourage modern visions and uses of its past. Rather than separating people, our understanding of cultures researched by scientists will attempt to see historical and contemporary commonalities and/or bridges between differences.
In this framework the EU project INCEPTION - Inclusive Cultural Heritage in Europe through 3D semantic modelling, funded under the call Reflective Societies: Cultural Heritage and European Identities, Advanced 3D modelling for accessing and understanding European cultural assets, is developing new research avenues in the field of heritage $3 \mathrm{D}$ data acquisition and modelling. Among different purposes, the project aims at the optimization of a 3D data acquisition protocol able to guide the process of digitization of cultural heritage, and innovation strategies to the three-dimensional modelling.

Thanks to a consortium of fourteen partners from ten European countries led by the Department of Architecture of the University of Ferrara, INCEPTION is able to balance research and business in different specific fields of interest of Cultural Heritage. The interdisciplinary consortium ranges from documentation and diagnostic skills, management, 3D acquisition technologies, development of hardware, software and digital platforms. The overall project aims at representation and dissemination of cultural heritage through ICT processes, analysis of semantic information to a wider and more extensive use of digital models, up to the market research and business strategies for the economic value of cultural heritage, a sector struggling because of the financial crisis.

The project, lasting four years, started in June 2015 and it is now approaching the second year of development; the first stage related to the enhancement in 3D acquisition processes has been accomplished by improving first of all integrated data capturing methodologies, while the enhancement of technologies is under development. In the meanwhile, the research is dealing with the creation of interoperable 3D models in H-BIM environment to be shared by an open standard platform.

\footnotetext{
* Corresponding author
} 
In order to maximize the impact, the INCEPTION project adopted a broad and a dedicated Stakeholder Panel, providing a significant panel discussion with experts in the field of Cultural Heritage not only scientifically but also directing research toward those strategies needed by "end users" and institutions. The aim is to increase knowledge, enhancement and dissemination through 3D digital models in order to promote the inclusiveness and accessibility of European cultural heritage. The members of the dedicated Stakeholder Panel provided demonstration cases, supporting the consortium in data collection. Outcomes from these pilots will be deployed through either on-site or off-site applications and demonstration activities.

\subsection{The INCEPTION project}

The INCEPTION project main aim is focused on innovation in 3D modelling of cultural heritage through an inclusive approach for time-dynamic 3D reconstruction of heritage sites and on the possibility to create an inclusive understanding of European cultural identity and diversity by stimulating and facilitating collaborations across disciplines, technologies and sectors.

Within this overall structure, the project is divided into five main steps: setting up of a common framework and knowledge management; advancement into the integrated 3D data capturing methodology; semantic modelling for Cultural Heritage buildings in H-BIM environment; development of the INCEPTION platform; deployment and valorization through different on-site and off-site applications for a wide range of users.

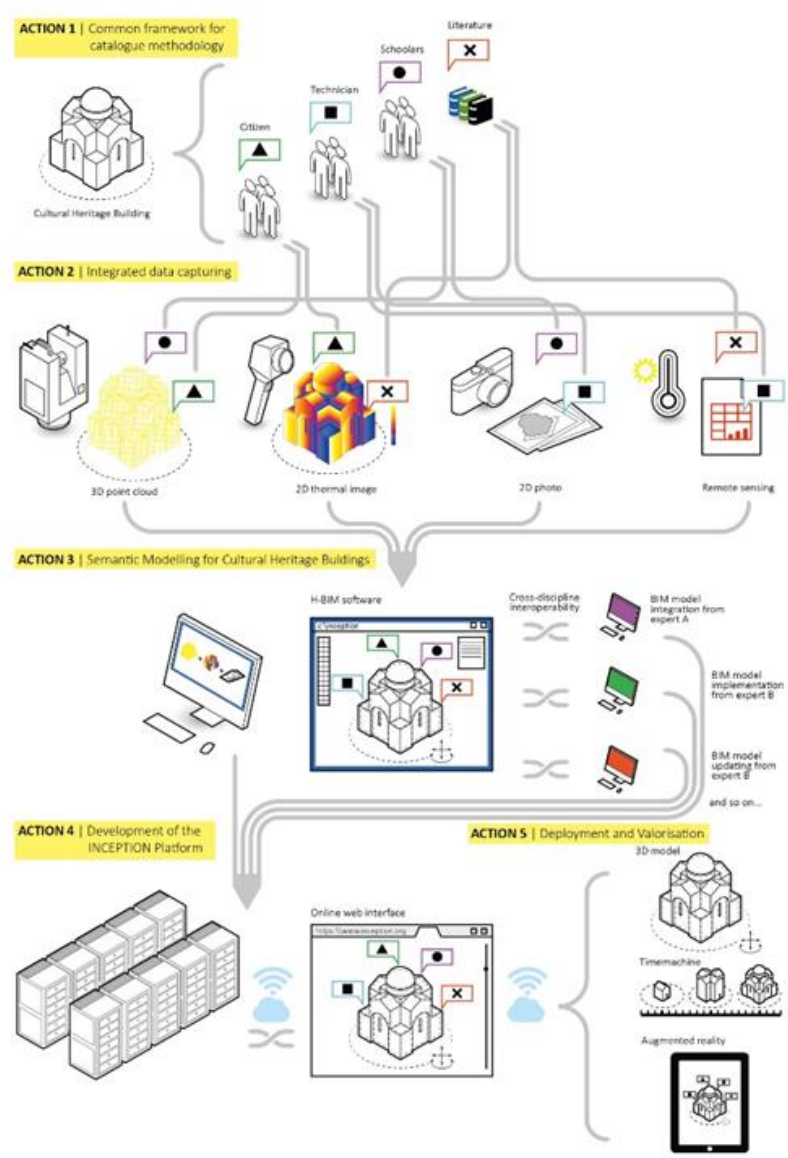

Figure 1. Scheme of the overall project concept and approach divided into five main Actions
The first step is related to the development of a common framework for catalogue methodology, basically by mapping the stakeholder' knowledge demands and targeted implementations by scholars, technicians, citizens and governments through the identification of key requirements that contribute to meet Europe's societal objectives related to Cultural Heritage (Di Giulio et al., 2016).

The second action is related to the integrated $3 \mathrm{D}$ data capturing, both as methodological procedure and optimized workflow specification by developing the INCEPTION data acquisition protocol, and by the enhancement of functionalities, capabilities and cost-effectiveness of data capturing technologies and documentation instruments (Maietti et al., 2016). Experimentation for an enhanced 3D data capturing system equipped with a new firmware optimized for cultural heritage application, addressed to an easier and more accessible data processing and modelling, will be accomplished during the third year of the project, putting in action the protocol during the survey procedures of the demonstration cases.

The third step is focused on the identification of the Cultural Heritage buildings semantic ontology and data structure for information catalogue. Integration of semantic attributes with hierarchically and mutually aggregated 3D digital geometric models is set up for managing heritage information (Ioannides et al., 2014).

The fourth action is the development of the INCEPTION Semantic Web Platform. The interoperable Semantic Web HBIM Platform will allow achieving the widest accessibility and interoperability, the use of three-dimensional models by researchers from different disciplines and non-expert users (Maravelakis et al., 2013), minimizing the difficulties of interaction with these kind of data, now accessible only by experts through the use of different software.

Deployment and Valorization will start by developing a wikilike feature to enable the sharing and enrichment of the information and interpretation of the models by users; the 3D models will be delivered in different Apps for numerous of purposes. The "browsing and query interface" will be defined allowing contributions by researchers and experts that actually do not deal with 3D data, and enabling a wide and easy access to the data by citizens, non-expert users and public at large.

\subsection{Complex heritage architectures}

INCEPTION deals with heritage "spaces" (complex architectures and sites), one of the most important "container" of cultural expressions in the evolution of the concept of European identity. The proposed integrated approach investigates the potential of spaces in order to create new cultural connections and awareness; the architecture is an outstanding example of the multi-layered conceptual dimension of European heritage.

The 3D survey of heritage architectural space needs a common protocol for data capturing and related enhancement of functionalities, capabilities and cost-effectiveness of technologies and documentation instruments (Zlota et al., 2014). The protocol considers the uniqueness of each site, quality indicators, time-consumption, cost-effectiveness, data accuracy and reliability, additional data and semantic proprieties to be recorded for heritage applications, adaptability to different sites with different historical phases.

The integration of digital data and the possibilities of re-use digital resources is an important challenge for protection and conservation of the historic buildings as well as for an efficient management in the long term (Stylianidis \& Remondino, 2016). The need of a future re-use of such broad and descriptive source 
of measurement data demands new applications to facilitate information accessing collected in three-dimensional databases without compromising the quality and amount of information captured in the survey (Remondino, 2011).

The identification of the multi-function and multi-scale role of the model allows the exploitation of uneasy and complex resources (obtained by the collection of geometric shape and not just of the architectural and urban context) at different levels, over time and by different actors. Here it is the value of accessibility/affordability of the process that until now has been barely allowed to spatial scale but through a mere visual navigation often un-interpreted, an approach very far from the knowledge, understanding and conservative needs (Fröhlich, 2015). The combination of innovative methodologies and protocols, processes, methods and devices allows enhancing the understanding of European Cultural Heritage by means of 3D models bringing new knowledge, collaboration across disciplines, time and cost saving in develop and use of $3 \mathrm{D}$ digital models (Ioannides et al., 2016).

The architectural space becomes the foundations, the common core and the "connection" for the creation of a protocol for optimizing the $3 \mathrm{D}$ documentation of cultural heritage. The methodology set as a priority the unconventional features/geometries, unique and complex within Heritage, avoiding the "segmentation" of data acquired and facilitating data access and use through an inclusive approach.

\section{THE INCEPTION DATA ACQUISITION PROTOCOL}

\subsection{Concept and approach}

In order to face the main challenges related to $3 \mathrm{D}$ survey of complex architectures and to start solving the issue of the large amount of captured data and time-consuming processes in the production of 3D digital models, an Optimized Data Acquisition Protocol (DAP) has been set up. The purpose is to guide the processes of digitization of cultural heritage, respecting needs, requirements and specificities of cultural assets, by dealing with the following issues:

- time consuming processes and limited budget available for 3D documentation,

- accuracy of 3D models,

- complexity of the heritage documentation,

- integration of metadata and semantics into the 3D model,

- link the 3D object with other multimedia information such as images, structural analysis data, materials, preservation records, etc. (Gomes et al., 2014),

- archiving of 3D digital records using wide accepted standards.
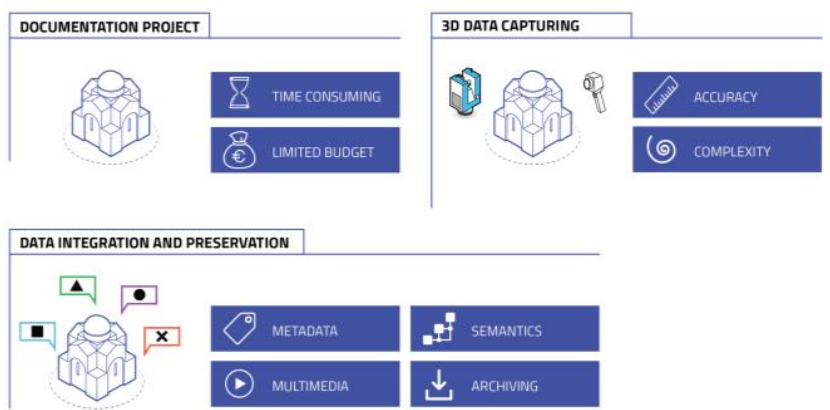

Figure 2. Main challenges in 3D documentation and conservation for Cultural Heritage related to the main workflow steps
The assessment and optimization of 3D data acquisition tools allow the improving of methodological and technological advancement for 3D data acquisition and development of procedural standards. The output is a methodological report for documenting Cultural Heritage by means of 3D data capturing. Within INCEPTION, the data acquisition protocol has been developed within a more general methodological procedure of heritage documentation (Stylianidis et al., 2011). Since every cultural asset is unique and requires survey, analysis and investigations "case by case", according to many different characteristics and to the main purposes of survey and documentation procedures, the protocol is set as flexible guidelines considering different kind of instruments and devices, different accuracies and levels of detail, etc., in addition to site specifications and the uniqueness of Cultural Heritage (Di Giulio et al., 2012).

The DAP can be followed during the planning and performing of a 3D laser scanner survey of Cultural Heritage, and it is referred to an architectural, archaeological, urban and site scale. It is also referred to data management (scan registration, data verification) data storage and archive. It is both a methodological procedure (Koussi et al., 2012) and an optimized workflow specification.

The main aims of the INCEPTION DAP are:

- to set up an optimized procedure, based on principles of simplicity and efficiency, for surveying heritage buildings and sites by using different 3D data capturing instruments;

- to provide a workflow for a consistent development of survey procedures for tangible cultural heritage and a set of instructions and guidelines for collecting, presenting and storing data;

- to provide a tool able to guide a 3D data capturing procedure able to generate 3D models accessible for a wide range of users; - to enhance the accuracy and efficiency of 3D data capturing by documentation and instruments integration;

- to support a cost effective and time saving procedure;

- to serve as the basis for the enhancement of functionalities of data capturing technologies and documentation instruments; - to close the gaps between technical fieldwork and modelling in $3 \mathrm{D}$ data capturing.

\subsection{Data Acquisition Protocol}

The DAP (Data Acquisition Protocol) provides a workflow for a consistent development of survey procedures for tangible cultural heritage and define a common background for the use of H-BIM across multiple building types and for a wide range of technical users (Pauwels et al., 2013). Furthermore, this protocol will be useful for any agency, organization or other institution that may be interested in utilizing survey procedures aimed at 3D H-BIM semantic models creation and their implementation for the INCEPTION platform. This protocol will be tested and further improved according to the specific test-bed procedures scheduled in INCEPTION research project. The DAP is intended to ensure uniformity in 3D digital survey for all the buildings that will be part of INCEPTION platform. This protocol considers a wide range of 3D data capturing instruments (Kadobayashi et al., 2004) because of multiple users and different techniques related to specific disciplines. Furthermore 3D survey instruments and techniques continue to evolve, and this protocol will continue to be reviewed and updated to reflect advances in industry technology, methodology and trends; in every case, the protocol application will ensure data homogenization between survey tailored to different requirements (Yen et al., 2011). 
The survey workflow was split into eight main steps that define specific requirements and their related activity indicators:

1. Scan Plan

2. Health and safety

3. Resolution Requirements

4. Registration mode

5. Control network

6. Quality control

7. Data control and verification

8. Data storage and archive

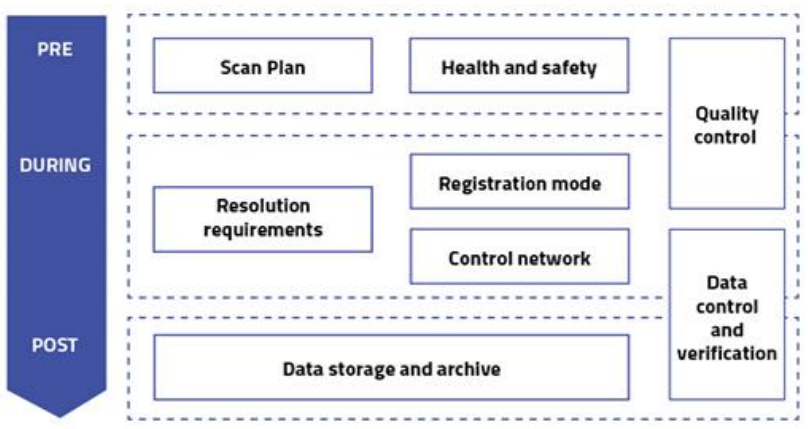

Figure 3. Workflow steps and activity indicators

Each step of the workflow must be intended as a set of questions that the technician, who is in charge to carry out the survey, should answer in order to pursue a correct data capturing. Those questions become a measuring system to verify the requirements of the survey, and the ability of finding the right answer define the level quality. On this assumption, every single question becomes an activity indicator that contributes to get a specific evaluation ranking. Not every activity indicator is always compulsory: if in the survey campaign only the minimum number of question find an answer, the capturing procedure will be classified in the lower ranking. Conversely, if each element is taken into account, the ranking will be the highest.

In case of directly measurable procedures, the specific activity indicator defines a range of accepted values. Instead, when alternative procedures are available, the protocol specifies their compliance with evaluation categories. For this purpose, there are four incremental categories defined as following:

B: This is the minimum evaluation category to be compliant with the INCEPTION Platform. It's intended to be used for very simple buildings or for the creation of low-detailed BIM model for digital reconstruction aimed at VR, AR and visualization purposes. In this case, the metric value of the model is less important than the morphological value.

A: This evaluation category is suitable for documentation purposes where the metric and morphological values are equivalent in term of impact on the survey that needs to be preliminary scheduled and designed. The registration process of 3D captured data can't be based only on morphological method but it should be improved by a topographic control network or GPS data.

A+: This evaluation category is the most suitable for preservation purposes because only the surveys compliant with this category could be a useful tool for restoration projects that need extremely correct metric data. From this surveys, BIM models as well as 2D CAD drawings till 1:20 scale are available. The project phase gets more importance than previous categories in order to schedule and manage the survey campaign and choose the right technical instruments to perform the data capturing. The management and the correction of metric errors are based on topographic techniques, in particular for what could concern the registration of different scan. The documentation phase will be developed organizing the information into Metadata and Paradata (Apollonio \& Giovannini, 2015). Elements of quality control are integrated into the process.

A++: This evaluation category is suitable for very complex buildings where the capturing process need to be documented and traced in order to get the maximum control on data or when monitoring process developed in a non-continuous time span take place. The A++ category could be useful even if different teams of technicians work together, simultaneously or in sequence, with different capturing instruments and different accuracies. The A++ category allows to analyse how a survey has been performed in every single phase: moreover this capability allows to integrate a survey in different times.

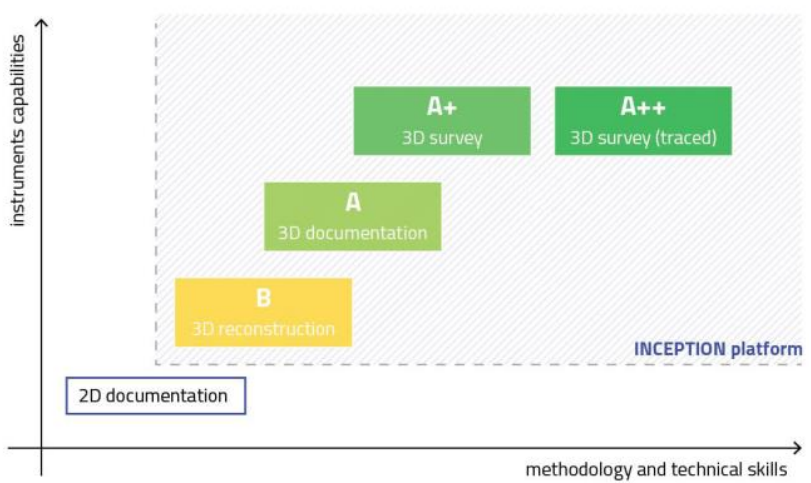

Figure 4. Evaluation categories

A direct correlation between evaluation category and type of building or deployment purposes could be identified. Indeed, complex buildings or advanced deployment purposes need a higher ranked capturing procedure. At the same time, it's necessary to point out that a further standardization should be avoided because every building or site is different from another and there is an increasing number digital data deployment.

\section{ASSESSMENT OF THE DATA ACQUISITION PROTOCOL}

\subsection{Analysis of benefits and added values by DAP}

In order to understand the impact of the INCEPTION DAP, a specific evaluation grid has been arranged, starting from the usual three standard features of quality, time and cost.

Each key feature for the evaluation of benefits and added values (Eppich \& Garcia Grinda, 2015) is specifically addressed looking at the main aims of the INCEPTION project. Since the evaluation process considers the point of view of the end users, that could be either technician or not, features have been developed as below:

- Quality can be evaluated as the reliability of the survey;

- Time can be evaluated as the usability of the survey;

- Cost can be evaluated as the effectiveness of the survey. 
Even if the accuracy and the precision are key factors for technicians that are performing the survey (Böhler et al., 2003), they are strictly connected with the purpose of the survey and for this reason using them for the evaluation of a survey quality becomes impossible, in particular from the point of view of an end user. The quality of a survey could be better described as the capability to be compliant with standards and ensure a long term support (Bryan et al., 2004). For this reason, key features of a reliable survey are:

- Survey maintenance: possibility to constantly update a survey database during a daily use of it for ordinary purposes, enriching it with new information of minor changes;

- Survey integration: possibility to perform major updates and upgrades of a survey, adding new part of a building or a site, previously not included in it, or performing more accurate survey of already existing parts;

- Tech obsolescence: because the hardware and the data management software are evolving faster and faster, applying strategies to avoid technical obsolescence became a key feature to ensure the survey reliability.

The measurement of benefits in term of time consumption could be performed taking into consideration the usability of the survey. More usable a delivered survey is, more time could be saved by the end users that will deal with it. One of the main aims of the procedure, indeed, is the ability of saving time in the processing phase The Data Acquisition Protocol and the adoption of a shared standard between suppliers and end users can bring a strong added value in terms of easy usability. For this reason, key features of a usable survey are:

- Common procedure: in order to ensure the full understanding of the output;

- Collaboration tools: in order to possible data creation by different teams at different times.

The cost of a survey it is always depending by the final quality and time spent to perform it. For this reason, the measurement of the effectiveness could be a better parameter to consider in order to evaluate the added value. For this reason, key features of an effective survey are:

- On field flexibility: possibility to use different kind of instruments on filed in order to produce the right amount of qualitative data, without the use of too expensive and not necessary ones;

- Easy deployment: ability to easily use the same delivered survey data for different kind of deployment and direct application for multiple purposes;

- Easy understanding: ability to easily read and understand data delivered with the survey from a low-skilled non-technician end user.

In order to measure benefits and added values by INCEPTION DAP, typical survey and documentation processes in cultural heritage field have been categorized in order to manly perform a grouping of an infinite number of different single and specific cases. The main connections between survey categories and DAP evaluation categories have been identified, and the DAP split in three on the basis of requirements that are needed to reach a better evolution category according to reliability, usability and effectiveness.

\subsection{Achieved results}

The definition of common framework for documentation and survey of European tangible cultural asset can take advantage by the assessment of the Data Acquisition Protocol.
Furthermore, following this assessment, it is possible to understand which are the main inputs to standardization in 3D data acquisition. Indeed, through a preliminary aggregation of data into ternary plots, it's easy to understand in which direction is possible to address the standardization process compared to the added value required. Below graphs are inserted.

\section{TO REACH EVALUATION CATEGORY A FROM B}

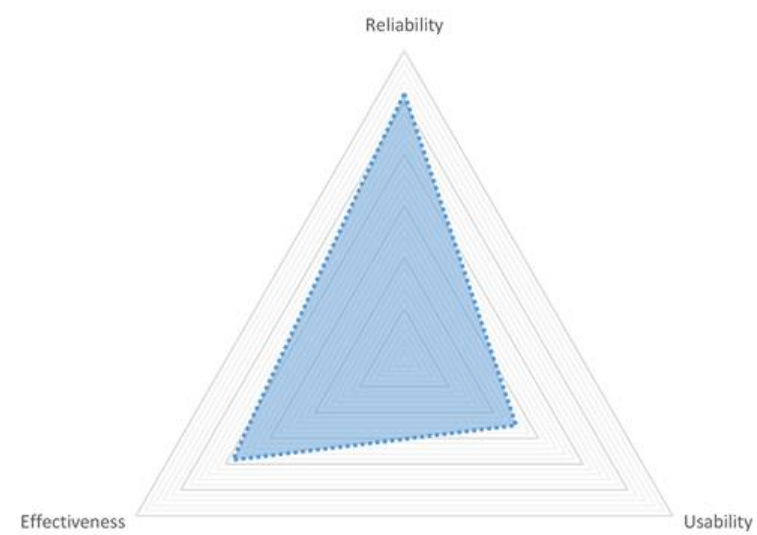

TO REACH EVALUATION CATEGORY A+ FROM A

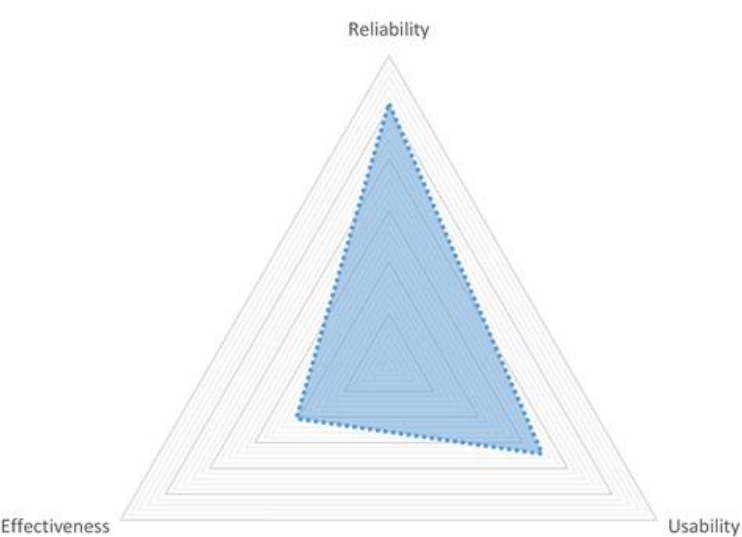

TO REACH EVALUATION CATEGORY A++ FROM A+

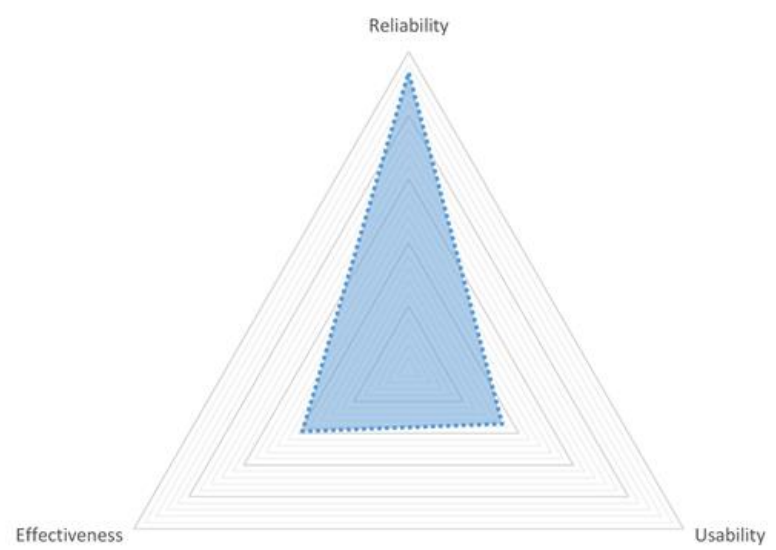




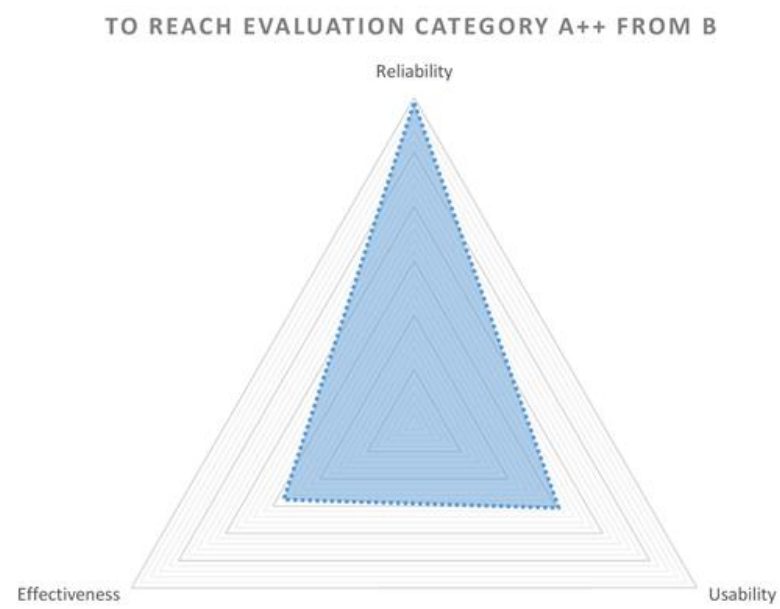

Figure 5. Data aggregation for the assessment of evaluation categories

From the graphical analysis of ternary plots results, it emerges clearly that:

- every incremental evaluation category strongly enhances the reliability of the survey and documentation;

- reaching the A category (from B) is also a great enhancing for the effectiveness of the results;

- reaching the A+ category (from A) is also a great enhancing for the usability of the results;

- reaching the A++ category (from $\mathrm{A}+$ ) is the greatest optimization of reliability;

From the overlay of the 3 different diagrams (normalized on the same base-value), it's also possible to state that easy reachability of the A category (starting from) but also a significant effort needed to get to the following one (A+).

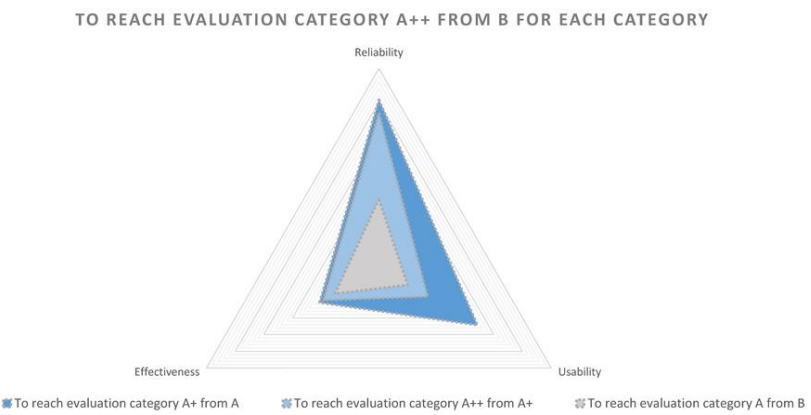

Figure 6. Data aggregation for each category

The A category can represent a widespread enhancement of the survey and documentation practice baseline and can be easily reached with relative low efforts. At the same time A+ is still the greatest improvement and allows to use survey results for purposes with a high level of requirements. Finally, the A++ category is the maximization of the reliability as the capability to be compliant with standards and ensure a long term support. On the other hand, thanks to the comprehension of advancement made possible by the protocol adoption, it will be possible, in future developments of this research, to focus on the integration of low-end data capturing instruments. The photo recognition and the creation of $3 \mathrm{D}$ models from a large set of photos are subjects where large steps have been made in the past years and if not now in the near future, 3D models will be easily produced typically from cultural heritage objects where many pictures from different angles are normally available (Koutsoudisa et al., 2014).

\section{CONCLUSIONS}

The INCEPTION project shows how the DAP allows to use the 3D data over time, from different skills, as well as a useful 3D digital data management toward H-BIM. Nevertheless, INCEPTION focuses, in further development steps, on open standards like E57 for point clouds and open standard IFC for semantic BIM data managed by Semantic Web-based technology to enable a long-term open access and interrelation of all available data.

INCEPTION innovation in 3D modelling applied to Cultural Heritage starts from the generation of high quality models, and therefore from an effective data capturing procedure. The DAP has been developed within a more general methodological procedure of heritage documentation. These guidelines will be gradually developed and updated during the project progress. Since every cultural asset is unique and requires analysis and investigations "case by case", the protocol is set as a methodological report and flexible guidelines considering site specifications and the uniqueness of Cultural Heritage.

Moreover, focusing on needs and requirements of technicians and non-technical users of heritage documentation, DAP assessment allow the of benefits from the application of standard procedures in Cultural Heritage field, starting from the assessment of different types of 3D survey and documentation (features, precision, speed, safety, area or range, environment), results, post processing and usage.

The definition of reliability, usability and effectiveness of the 3D survey better specifies the three standards features of quality, time, cost, in relation to the evaluation categories.

\section{ACKNOWLEDGEMENTS}

The project is under development by a consortium of fourteen partners from ten European countries led by the Department of Architecture of the University of Ferrara. Academic partners of the Consortium, in addition to the Department of Architecture of the University of Ferrara, include the University of Ljubljana (Slovenia), the National Technical University of Athens (Greece), the Cyprus University of Technology (Cyprus), the University of Zagreb (Croatia), the research centers Consorzio Futuro in Ricerca (Italy) and Cartif (Spain).

The clustering of small medium enterprises includes: DEMO Consultants BV (The Netherlands), 3L Architects (Germany), Nemoris (Italy), RDF (Bulgaria), 13BIS Consulting (France), Z + F (Germany), Vision and Business Consultants (Greece).

The INCEPTION project has been applied under the Work Programme Europe in a changing world - inclusive, innovative and reflective Societies (Call - Reflective Societies: Cultural Heritage and European Identities, Reflective-7-2014, Advanced 3D modelling for accessing and understanding European cultural assets).

This research project has received funding from the European Union's H2020 Framework Programme for research and innovation under Grant agreement no 665220. 


\section{REFERENCES}

Apollonio, F.I., Giovannini, E.C., 2015. A paradata documentation methodology for the Uncertainty Visualization in digital reconstruction of $\mathrm{CH}$ artifacts. SCIRES-IT-SCIentific Res. Inf. Technol. 5, 1-24.

Böhler, W., Bordas Vicent, M., \& Marbs, A., 2003. Investigating Laser Scanner Accuracy. The International Archives of Photogrammetry, Remote Sensing and Spatial Information Sciences, Vol. XXXIV, Part, 5, C15.

Bryan, P. G., Barber, D. M., \& Mills, J. P., 2004. Towards a standard specification for terrestrial laser scanning in cultural heritage-one year on. International Archives of the Photogrammetry, Remote Sensing and Spatial Information Sciences, 35(B7), 966-971.

Di Giulio R., Balzani M., Piaia E. and Zuppiroli M., 2012. European Protocols for methods, tools and techniques: new standard for 3D Laser Scanner. In: Roko Žarnic, Vlatka Rajcic, Barbara Vodopivec (editors), Heritage Protection. From documentation to interventions. Proceedings of the EU-CHIC International Conference on Cultural Heritage Preservation, University of Zagreb, Croatia, pp. 149-151.

Di Giulio R., Maietti F., Piaia E., 2016. 3D documentation and semantic aware representation of Cultural Heritage: the INCEPTION project. EUROGRAPHICS Workshop on Graphics and Cultural Heritage, pp. 195-198.

Eppich R., Garcia Grinda. J.L., 2015. Management Documentation Indicators \& Good Practices at Cultural Heritage Palces. The International Archives of the Photogrammetry, Remote Sensing and Spatial Information Science, Volume XL-5/W7, 2015. 25th International CIPA Symposium, Taipei, Taiwan.

Fröhlich C., Mettenleiter M., Held C., Blersch D. and Kurz S., 2015. Surveying by means of both 3D Geometry with HDR Colour and Thermal Imagery. Automatisierungstechnik. Issue 63, book 4, p. 279-285.

Gomes L., Regina O., Bellon P. and Silva L., 2014. 3D reconstruction methods for digital preservation of cultural heritage: A survey. In: Pattern Recognition Letters, Volume 50, 1 December 2014, pp. 3-14.

Ioannides, M., Fink, E., Moropoulou, A., Hagedorn-Saupe, M., Fresa, A., Liestøl, G., Rajcic, V., Grussenmeyer, P. (Eds.), 2016. Digital Heritage. Progress in Cultural Heritage: Documentation, Preservation, and Protection, 6th International Conference, EuroMed 2016, Springer Berlin Heidelberg.

Ioannides, M., Magnenat-Thalmann, N., Fink, E., Zarnic, R., Yen, A.-Y., Quak, E. (Eds.), 2014. Digital Heritage. Progress in Cultural Heritage. Documentation, Preservation, and Protection. Proceedings of the 5th International Conference, EuroMed, Springer Berlin Heidelberg.

Kadobayashi, R., Kochi, N., Otani, H., \& Furukawa, R., 2004. Comparison and evaluation of laser scanning and photogrammetry and their combined use for digital recording of cultural heritage. International Archives of the Photogrammetry, Remote Sensing and Spatial Information Sciences, 35(5), 401-406.
Koussi A, Karoglou M, Labropoulos K, Bakolas A, Moropoulou A., 2012. Integrated Documentation Protocols able to support decision-making process in Cultural Heritage protection. In: Roko Žarnic, Vlatka Rajcic, Barbara Vodopivec (editors), Heritage Protection. From documentation to interventions. Proceedings of the EU-CHIC International Conference on Cultural Heritage Preservation, University of Zagreb, Croatia, pp. 129-131.

Koutsoudisa A., Vidmarb B., Ioannakisa G., Arnaoutogloua F., Pavlidis G., Chamzasc C., 2014. Multi-image 3D reconstruction data evaluation. Journal of Cultural Heritage. 15 (2014) 73-79.

Maietti F., Ferrari F., Medici M., Balzani M., 2016. 3D Integrated Laser Scanner Survey and Modelling for Accessing and Understanding European Cultural Assets. In: Ruben Paul Borg, Paul Gauci, Cyril Spiteri Staines (Eds.), Proceedings of the International Conference "SBE Malta 2016. Europe and the Mediterranean: Towards a Sustainable Built Environment", Gutenberg Press, Malta, pp. 317-324.

Maravelakis, E., Konstantaras, A., Kritsotaki, A., Angelakis, D., Xinogalos, M., 2013. Analysing user needs for a unified 3D metadata recording and exploitation of cultural heritage monuments system. In: International Symposium on Visual Computing (pp. 138-147). Springer Berlin Heidelberg.

Pauwels, P., Bod R., Di Mascio D. and De Meyer R., 2013. Integrating building information modelling and semantic web technologies for the management of built heritage information. In: Digital Heritage International Congress (Digital Heritage), 2013 (Volume:1). Publisher: IEEE. pp. 481 - 488.

Remondino F., 2011. Heritage Recording and 3D Modeling with Photogrammetry and 3D Scanning. Remote Sensing, 3, pp. 1104-1138.

Remondino, F., Rizzi, A., 2010. Reality-based 3D documentation of natural and cultural heritage sites techniques, problems, and examples. Applied Geomatics, 2(3), $85-100$.

Stylianidis, E., Remondino, F. (Eds.), 2016. 3D recording, documentation and management of cultural heritage. Whittles Publishing, Dunbeath, Caithness, Scotland.

Stylianidis, E., Patias, P., Santana Quintero, M. (Eds.), 2011. CIPA Heritage Documentation: Best Practices and Applications. The ICOMOS \& ISPRS Committee for Documentation of Cultural Heritage, Volume XXXVIII-5/C19, Series 1, $2007 \& 2009$.

Yen, Y.N., Weng K.H., Cheng H.M., Hsu W.S., 2011. The Standard of Management and Application of Cultural Heritage Documentation Cultural Heritage Documentation. In: XXIIIrd Symposium CIPA, 2011.

Zlota, R., Bossea M., Greenopb K., Jarzaba Z., Juckesb E., Robertsa J., 2014. Efficiently capturing large, complex cultural heritage sites with a handheld mobile 3D laser mapping system. Journal of Cultural Heritage 15 (2014) 670-678. 